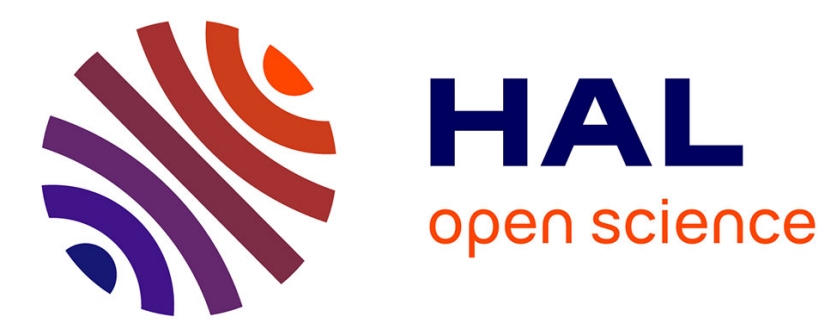

\title{
Silice UVCVD pour transistors MISFET autoalignés sur InP
}

\author{
J.L. Courant, P. Dimitriou, G. Post
}

\section{To cite this version:}

J.L. Courant, P. Dimitriou, G. Post. Silice UVCVD pour transistors MISFET autoalignés sur InP. Revue de Physique Appliquée, 1990, 25 (9), pp.935-939. 10.1051/rphysap:01990002509093500 . jpa00246257

\section{HAL Id: jpa-00246257 https://hal.science/jpa-00246257}

Submitted on 1 Jan 1990

HAL is a multi-disciplinary open access archive for the deposit and dissemination of scientific research documents, whether they are published or not. The documents may come from teaching and research institutions in France or abroad, or from public or private research centers.
L'archive ouverte pluridisciplinaire HAL, est destinée au dépôt et à la diffusion de documents scientifiques de niveau recherche, publiés ou non, émanant des établissements d'enseignement et de recherche français ou étrangers, des laboratoires publics ou privés. 
Classification

Physics Abstracts

73.40Q

\title{
Silice UVCVD pour transistors MISFET autoalignés sur InP
}

\author{
J. L. Courant, P. Dimitriou et G. Post \\ C.N.E.T., Laboratoire de Bagneux, 196 avenue Henri Ravera, 92220 Bagneux, France
}

(Reçu le 20 décembre 1989, accepté le 12 juin 1990)

\begin{abstract}
Résumé. - Nous utilisons un mode de dépôt de silice photochimique pour la réalisation d'un transistor MISFET sur substrat en phosphure d'indium, InP, à grille autoalignée. Cette technique nécessite l'emploi d'un recuit à haute température. Nous avons soumis ces films à des recuits rapides, sous lampes, dans une gamme de température de 300 à $900{ }^{\circ} \mathrm{C}$. Les analyses ont été faites par spectroscopie infrarouge par transmission, ellipsométrie spectroscopique, mesures de capacités de diodes MIS à $1 \mathrm{MHz}$, et courants de fuite. Nous avons constaté une déshydratation rapide, une amélioration notable de la résistivité et de la constante diélectrique. Il apparaît des charges fixes dans l'oxyde qu'un recuit à basse température permet d'éliminer. La densité de ces films est de 2,06. L'ellipsométrie révèle un léger excès de silicium $(1 \%)$. Les performances des premiers transistors restent modestes $(12 \mathrm{mS} / \mathrm{mm})$ mais avec une faible dérive $\left(<\mathrm{qq} \%\right.$ sur $\left.10^{4} \mathrm{~s}\right)$ à $V_{\mathrm{ds}}=0,1 \mathrm{~V}$ et $V_{\mathrm{gs}}=2,5 \mathrm{~V}$.
\end{abstract}

Abstract. - A photochemical technique for silica deposition is presented, in view of the realisation of selfaligned gate MISFET's on indium phosphide substrate. This technology requires a high temperature anneal. We submitted those films to rapid thermal annealing between 300 and $900{ }^{\circ} \mathrm{C}$. Analyses have been performed by infra-red transmission spectroscopy, spectroscopic ellipsometry, measurement of capacitance of MIS diodes at $1 \mathrm{MHz}$, and of leakage current. We found a significant dehydration, a strong improvement of the resistivity and of the dielectric constant. The fixed charges which appear in the oxide are eliminated by a low temperature anneal. The density of these films is 2.06. Ellipsometry revealed a slight excess of silicon (1\%). The transconductance of the first transistors is $12 \mathrm{mS} / \mathrm{mm}$, with a low current drift (a few \% over $10^{4} \mathrm{~s}$ ) at $V_{\mathrm{ds}}=0.1 \mathrm{~V}$ and $V_{\mathrm{gs}}=2.5 \mathrm{~V}$.

\section{Introduction.}

L'amélioration des télécommunications par fibre optique réclame des composants d'extrémité rapides. Afin de répondre à cette demande l'intégration opto-électronique semble s'imposer. Le substrat en phosphure d'indium est un matériau adapté aux longueurs d'onde utilisées (entre 1,3 et 1,55 $\mu \mathrm{m}$ ), en raison de l'épitaxie avec accord de maille des couches actives de lasers et de photodétecteurs. Il a en tant que matériau actif de transistors l'atout d'une forte mobilité électronique. Nous avons étudié un transistor à effet de champ à grille isolée, MISFET. Nous faisons appel à une technique de dépôt d'isolant, dont la maîtrise est essentielle, puisque de la qualité de l'interface silice-InP dépendent les caractéristiques de fonctionnement du transistor. L'autoalignement de la source et du drain réalisés par implantation de $\mathrm{Si}$, dopant $\mathrm{n}$, masqué par la grille, entraîne un positionnement parfait et reproductible des differentes zones actives. La structure MIS doit supporter lé recuit rapide de l'implantation, aussi la grille estelle fabriquée en métal réfractaire à base de tungstène. L'isolant retenu est la silice déposée à basse température par photolyse (UVCVD). Le problème qui nous préoccupe est le comportement de cet isolant fabriqué à basse température lors des recuits d'implantation $\left(>600^{\circ} \mathrm{C}\right)$.

\section{Moyens d'analyse.}

Des analyses en transmission infrarouge ont été effectuées à l'aide d'un appareil de spectroscopie à transformée de Fourier (Perkin-Elmer 1760). L'emploi d'un substrat de référence identique à celui de l'échantillon permet d'éliminer le bruit de fond et le signal du substrat. Dans la gamme de nombre d'onde $400 \mathrm{~cm}^{-1}$ à $4000 \mathrm{~cm}^{-1}$, cette mesure révèle la présence de nombreux groupes d'atomes par absorption des photons aux énergies de vibration de leurs liaisons (Tab. I) [1]. 
Tableau I. - Récapitulatif des pics d'absorption observés sur les silices UVCVD fabriquées à $170{ }^{\circ} \mathrm{C}$ $[1,4]$.

[Absorption peaks observed with UVCVD silica made at $\left.170{ }^{\circ} \mathrm{C}[1,4].\right]$

\begin{tabular}{|c|c|c|}
\hline $\begin{array}{c}\text { Position } \\
\mathrm{cm}^{-1}\end{array}$ & Vibration & Observation \\
\hline 800 & $\mathrm{Si}-\mathrm{O}$ & \\
\hline 880 & $\mathrm{SiH}_{2}$ & A fort débit de $\mathrm{SiH}_{4}$ \\
\hline 940 & $\mathrm{Si}-\mathrm{OH}$ & \\
\hline 1065 & $\mathrm{Si}-\mathrm{O}$ & Vibration longitudinale \\
\hline 1630 & $\mathrm{~N}=\mathrm{O}$ & A faible débit de $\mathrm{SiH}_{4}$ \\
\hline 2200 & $\mathrm{Si}-\mathrm{H}$ & \\
\hline 3300 & $\mathrm{H}$ & \\
\hline 3660 & $\mathrm{SiO}-\mathrm{H}$ & Large bande d'absorption \\
\hline
\end{tabular}

L'ellipsométrie spectroscopique entre 1,5 et $5 \mathrm{eV}$, par la mesure des paramètres $\Delta$ et $\Psi$, puis simulation numérique permet d'évaluer, sous certaines hypothèses, la composition et la porosité des couches [3].

Des mesures électriques de capacités à $1 \mathrm{MHz}$, et de courants de fuite ont été faites sur des capacités MIS, sur des substrats de silicium et d'InP. Elles permettent de connaître la densité de pièges à l'interface, la constante diélectrique (mesurée à partir de la capacité d'oxyde de diodes MIS $\mathrm{SiO}_{2} / \mathrm{Si}$ ), et la résistivité du film (à un champ de $500 \mathrm{kV} / \mathrm{cm}$ ).

\section{Dépôt/échantillons.}

Les échantillons sont maintenus à une température de $180^{\circ} \mathrm{C}$, par une rampe de lampes infrarouges. Une série de lampes au mercure permet la photolyse des molécules de silane et de protoxyde d'azote, en présence d'atomes de mercure dans la phase gazeuse, la pression dans le réacteur étant maintenue à 1 Torr et le rapport des flux $\mathrm{N}_{2} \mathrm{O} / \mathrm{SiH}_{4}$ à $30 / 1$. Les vitesses de dépôts sont de 120 à $150 \AA / \mathrm{min}$ [2].

Les recuits rapides ont été effectués afin de simuler les recuits à haute température $\left(>600^{\circ} \mathrm{C}\right)$ nécessaires à l'activation des impuretés dopantes implantées. Il s'agit ici d'un four à lampes commandé par un automate programmable. Après pompage, l'enceinte est sous balayage permanent d'argon hydrogéné $(10 \%)$. Un thermocouple relié à une table traçante mesure la température de l'échantillon. Le temps de montée est de 10 secondes suivi d'un palier de 10 secondes à la température choisie.

Les électrodes des diodes MIS sont réalisées par la technique du lift-off. La métallisation de Ti/Au se fait au canon à électron. Les motifs sont de trois diamètres différents. Ces tailles nous permettent de nous assurer d'un bon contact électrique lors des mesures, par la linéarité des mesures de capacité.

\section{Résultats.}

Après dépôt.

Dans les spectres de transmission infrarouge nous reconnaissons les 2 bandes typiques correspondant aux liaisons $\mathrm{Si}-\mathrm{O}$, à $800 \mathrm{~cm}^{-1}$ vibration transversale, à $1065 \mathrm{~cm}^{-1}$ vibration longitudinale [1]. La position et l'intensité de ce dernier pic révèlent, comparé à un spectre d'oxyde natif de silicium préparé à $1100{ }^{\circ} \mathrm{C}$, un manque de liaison Si-O. Ce phénomène est à relier avec une mesure de densité $(2,06$ au lieu de 2,2).

La gamme de nombre d'onde $3000 \mathrm{~cm}^{-1}$ à $4000 \mathrm{~cm}^{-1}$ révèle la présence d'une importante quantité de liaisons $\mathrm{O}-\mathrm{H}$. Une large bande centrée vers $3300 \mathrm{~cm}^{-1}$ est attribuée aux molécules $\mathrm{H}_{2} \mathrm{O}$. Un pic plus fin centré vers $3660 \mathrm{~cm}^{-1}$ est attribué aux groupements $\mathrm{Si}-\mathrm{OH}$, ainsi que celui à $940 \mathrm{~cm}^{-1}$. Leur présence semble inhérente aux températures de dépôt employées. Une variation du flux de silane, à température constante, réduit de manière significative l'amplitude des pics associés à ces groupements d'atomes, mais au détriment de l'apparition de composés tels que NO $\left(1630 \mathrm{~cm}^{-1}\right)$ à faible débit de $\mathrm{SiH}_{4}$ ou $\mathrm{Si}-\mathrm{H}\left(2200 \mathrm{~cm}^{-1}\right)$, et même $\mathrm{SiH}_{2}$ $\left(880 \mathrm{~cm}^{-1}\right)$, à fort débit de $\mathrm{SiH}_{4}$. Des mesures quantitatives ont conduit à évaluer la présence d' $\mathrm{H}_{2} \mathrm{O}$ à environ $10 \%$ et de $\mathrm{SiOH}$ à environ $6 \%$ en masse [4].

Les spectres d'ellipsométrie ont été interprétés en supposant une couche de mélange hétérogène de $\mathrm{Si}$ amorphe et de $\mathrm{SiO}_{2}$ idéale. Un léger excès de silicium évalué à $1 \%$ ou $2 \%$ est alors obtenu. L'influence des caractéristiques de la silice employée dans la simulation est importante. La valeur de $1 \%$ est obtenue pour une silice de densité 2,2 , et celle de $2 \%$ pour une silice de densité 2,06 , qui semble plus proche de notre cas.

Les mesures électriques ont déjà fait l'objet de publications [3]. Des mesures de densité d'interface, par la méthode de Terman, ont donné des valeurs de $10^{11}$ à $10^{12} \mathrm{eV}^{-1} \mathrm{~cm}^{-2}$, avec un faible hystérésis, $<1 \mathrm{~V}$.

Les mesures RBS ont montré que les atomes de $\mathrm{Hg}$ servant à l'activation de la réaction étaient présents à une concentration inférieure à $10^{17}$ atomes $/ \mathrm{cm}^{3}$. 


\section{Recuits rapides.}

Nous avons constaté la disparition des pics d'absorption des liaisons $\mathrm{OH}$, aux fortes valeurs de nombre d'onde, à partir de températures de $500{ }^{\circ} \mathrm{C}$. Ce type de recuit semble efficace en ce qui concerne la déshydratation des films, sans pour autant que nous puissions en conclure qu'elle soit totale, compte tenu de la sensibilité des mesures $(<0,5 \%)$. Nous n'avons pas constaté d'évolution significative du pic Si-O à $1065 \mathrm{~cm}^{-1}$ (Fig. 1), alors que des recuits à basse température $\left(250^{\circ} \mathrm{C}\right)$ de plusieurs heures, s'ils déshydratent peu le film, amènent une augmentation de l'amplitude de ce pic. Nous rapprochons ces faits des mesures de densité qui n'ont pas montré une réelle densification de la couche. La perte de masse en $\mathrm{H}_{2} \mathrm{O}$ semble compensée par la perte d'épaisseur. Alors qu'une restructuration du film, sans perte de masse, aurait entraîné une densité plus élevée.

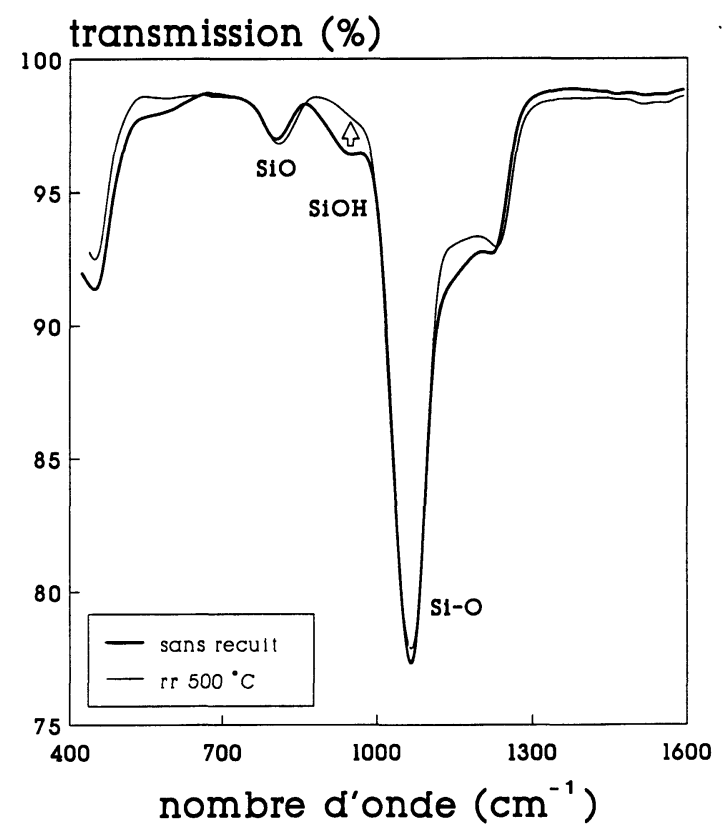

Fig. 1. - Spectre infrarouge entre $400 \mathrm{~cm}^{-1}$ et $1600 \mathrm{~cm}^{-1}$ avant (trait fin) et après (trait gras) recuit flash à $500^{\circ} \mathrm{C}$, $10 \mathrm{~s}$.

[Infra-red spectrum, between $400 \mathrm{~cm}^{-1}$ and $1600 \mathrm{~cm}^{-1}$, before and after Rapid Thermal Anneal at $500{ }^{\circ} \mathrm{C}, 10 \mathrm{~s}$.]

Nous avons remarqué un fort décalage des caractéristiques de capacité vers les tensions négatives. Ces charges positives apparaissent dès $300{ }^{\circ} \mathrm{C}$ et se stabilisent au-delà de $600{ }^{\circ} \mathrm{C}$ à une valeur de décalage d'environ $-15 \mathrm{~V}$. La densité de ces charges est estimée à $N_{f} \approx 10^{18} \mathrm{~cm}^{-3}$. Ces nombreux défauts sont guéris par un recuit à basse température, sous atmosphère d'oxygène sec, de longue durée (Fig. 2).

Nous attribuons ces charges aux liaisons pendantes de silicium laissées libres par l'éviction d'atomes

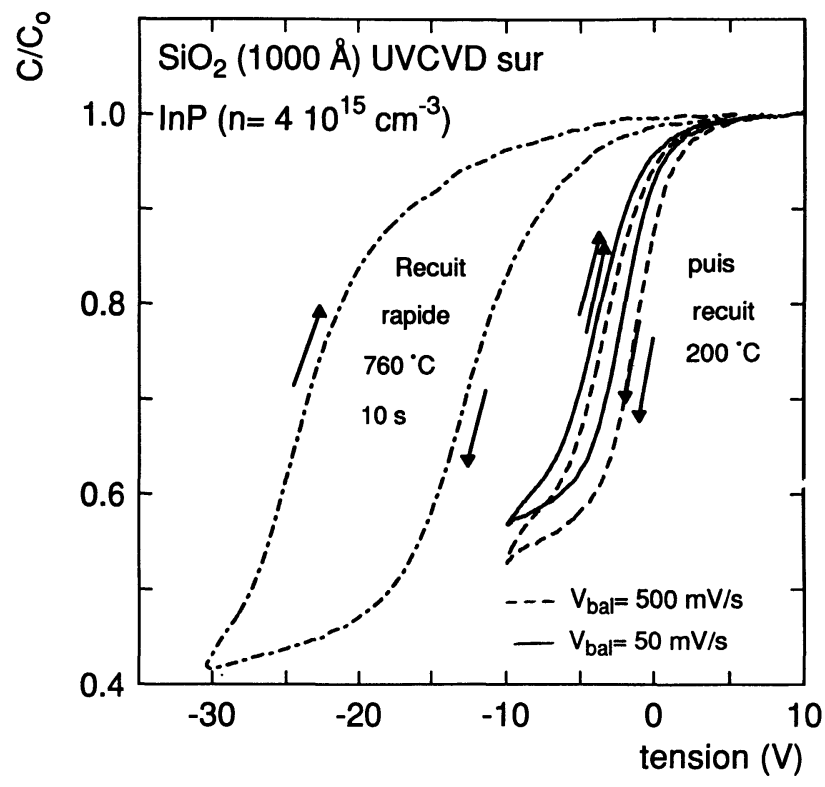

Fig. 2. $-C-V$ à $1 \mathrm{MHz}$ de diodes $\mathrm{MIS} \mathrm{SiO}_{2}$ (UVCVD)/InP après recuit rapide $760{ }^{\circ} \mathrm{C} 10 \mathrm{~s}$ (vitesse de balayage : $\left.V_{\text {bal }}=500 \mathrm{mV} / \mathrm{s}\right)$ puis recuit de guérison à $200{ }^{\circ} \mathrm{C}$ $\left(V_{\text {bal }}=500 \mathrm{mV} / \mathrm{s}\right.$ et $\left.50 \mathrm{mV} / \mathrm{s}\right)$.

[ $C-V$ at $1 \mathrm{MHz}$ of MIS diodes $\mathrm{SiO}_{2}$ (UVCVD)/InP after rapid thermal anneal at $760{ }^{\circ} \mathrm{C}, 10 \mathrm{~s}$ (sweep rate : $V_{\text {bal }}=500 \mathrm{mV} / \mathrm{s}$ ), and stabilization anneal at $200{ }^{\circ} \mathrm{C}$ $\left(V_{\text {bal }}=500 \mathrm{mV} / \mathrm{s}\right.$ and $\left.50 \mathrm{mV} / \mathrm{s}\right)$.]

d'hydrogène sans formation de liaison $\mathrm{SiO}$, qui ne se forment que lors de recuits prolongés comme nous l'avons vu précédemment. Nous avons observé l'évolution de la constante diélectrique, qui d'une valeur très élevée, se stabilise à une valeur de 4 vers $600{ }^{\circ} \mathrm{C}$ (Fig. 3). Ce comportement peut être expliqué par la présence de liaisons $\mathrm{O}-\mathrm{H}$ très facilement polarisables. La valeur finale reste légèrement supérieure à 3,9 , valeur nominale de la silice, ce que nous attribuons au SiOH résiduel, qui est aussi présent dans des films fabriqués à plus haute température. Les mesures de courant de fuite font apparaître une amélioration spectaculaire de la résistivité qui passe de quelque $10^{13} \Omega . c m$ à $10^{15} \Omega . c m$ (Fig. 4). Les mesures d'ellipsométrie après recuit rapide confirment la présence d'un léger excès de silicium. La valeur un peu faible atteinte par l'indice vers $600{ }^{\circ} \mathrm{C}$, malgré la stœchiométrie riche en silicium confirme que la densité de ces couches reste inférieure à celle de la silice thermique (Fig. 5).

\section{Composants.}

Les premiers transistors réalisés avec la technologie de l'autoalignement, avec une grille en nitrure de tungstène déposée par pulvérisation réactive, ont présenté une tenue mécanique satisfaisante sous recuit rapide. En effet le dégazage de la silice UV et les variations de contraintes de la couche de métal réfractaire conduisent facilement à l'apparition de 


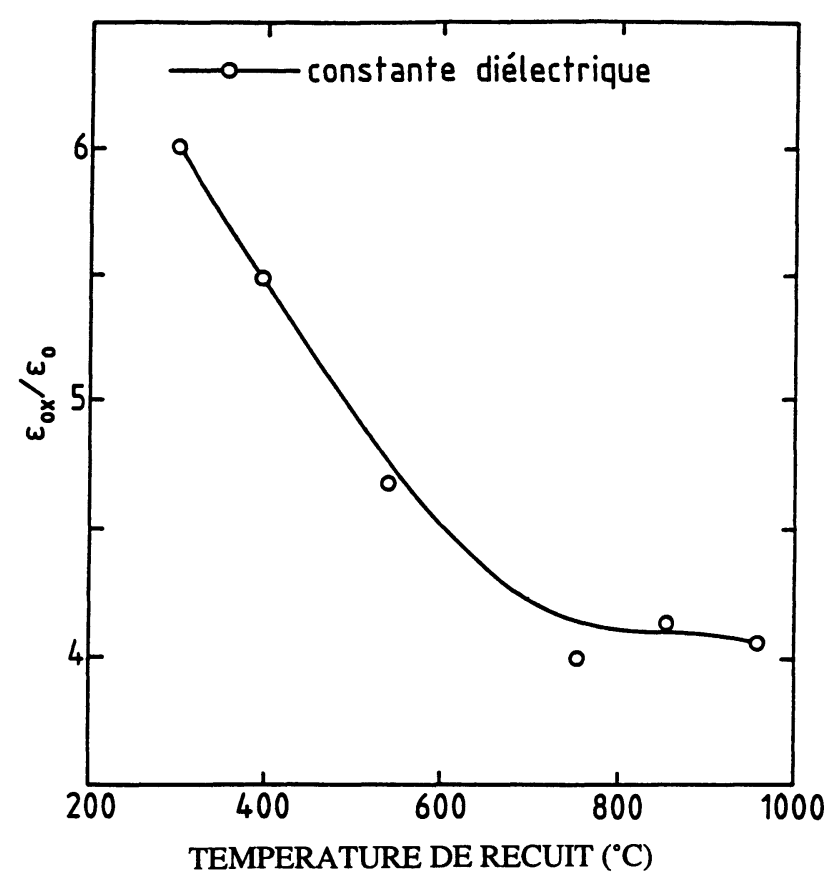

Fig. 3. - Evolution de la constante diélectrique des films de $\mathrm{SiO}_{2}$ avec la température du recuit rapide (10 s).

[Variation of dielectric constant of $\mathrm{SiO}_{2}$ films with temperature of rapid thermal anneal (10 s).]

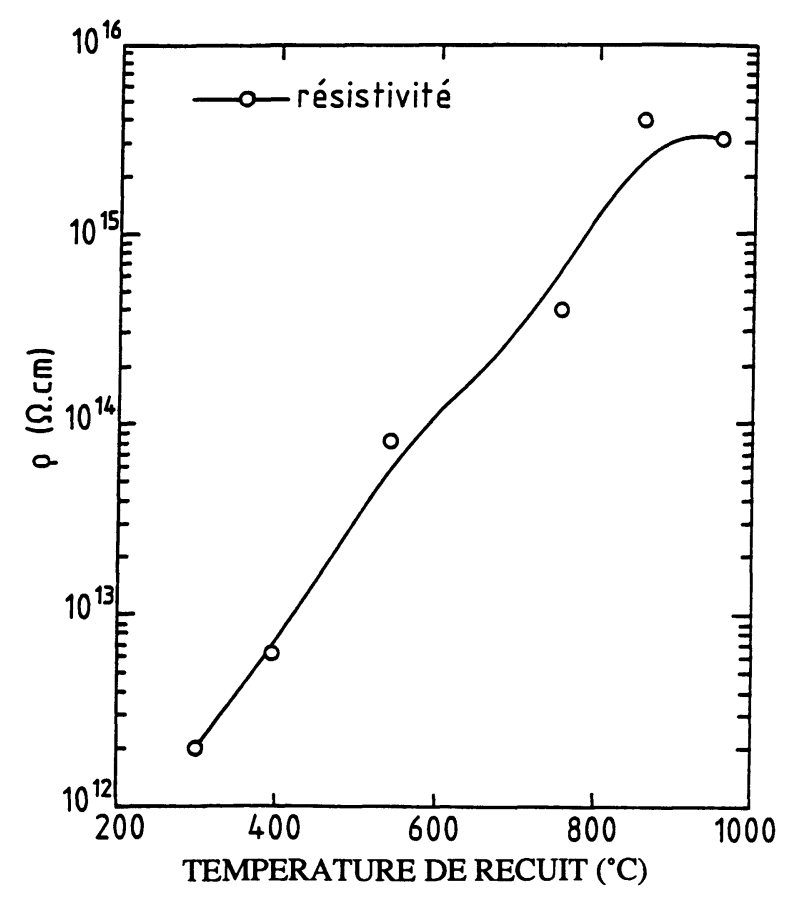

Fig. 4. - Evolution de la résistivité des films de $\mathrm{SiO}_{2}$ avec la température du recuit rapide (10 s).

[Variation of resistivity of $\mathrm{SiO}_{2}$ films with temperature of rapid thermal anneal (10 s).]

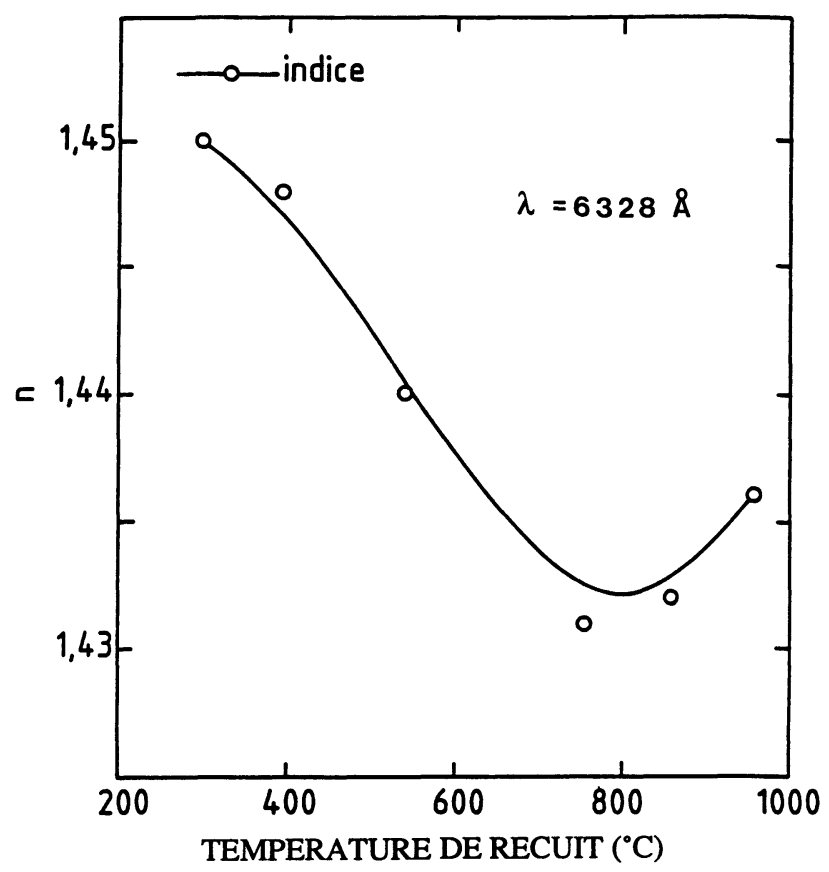

Fig. 5. - Evolution de l'indice des films de silice, mesuré à $\lambda=6328 \AA$, avec la température du recuit rapide (10 s).

[Variation of index (at $\lambda=6328 \AA$ ) of $\mathrm{SiO}_{2}$ films with temperature of rapid thermal anneal $(10 \mathrm{~s})$.]

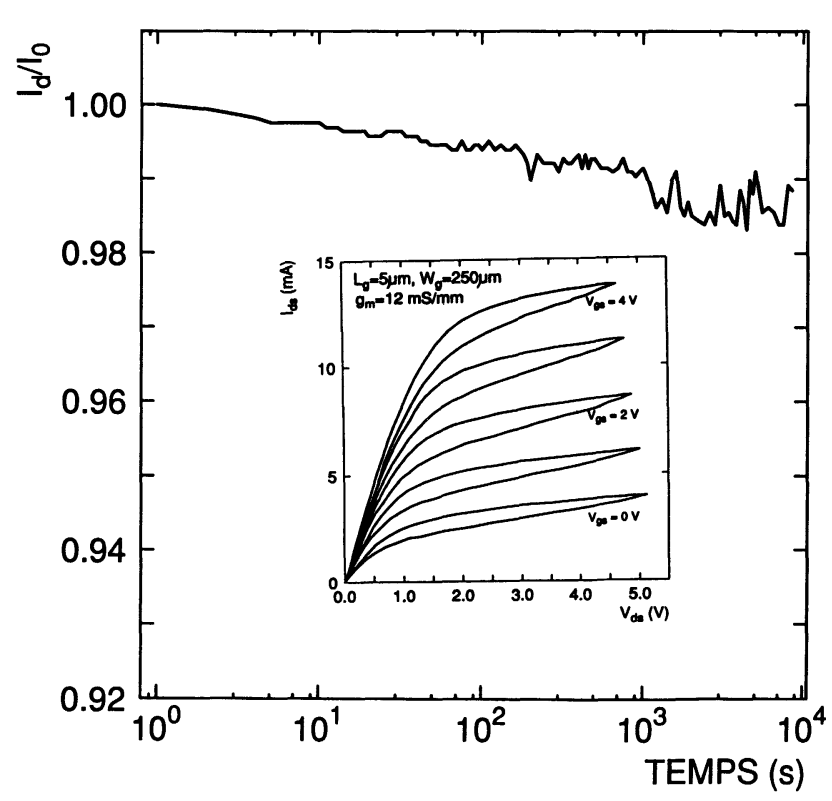

Fig. 6. - Dérive du courant $I_{\mathrm{ds}}$ en régime linéaire $\left(V_{\mathrm{ds}}=0,1 \mathrm{~V}, V_{\mathrm{gs}}=2,5 \mathrm{~V}\right)$, et réseau de caractéristiques $I_{\mathrm{ds}}\left(V_{\mathrm{ds}}, V_{\mathrm{gs}}\right)$ d'un transistor MISFET sur InP à grille réfractaire $\left(\mathrm{WN}_{x}\right), L_{\mathrm{g}}=5 \mu, W_{\mathrm{g}}=250 \mu$.

[ $I_{\mathrm{ds}}$ current drift at $V_{\mathrm{ds}}=0,1 \mathrm{~V}, V_{\mathrm{gs}}=2,5 \mathrm{~V}$, and characteristics $I_{\mathrm{ds}}\left(V_{\mathrm{ds}}, V_{\mathrm{gs}}\right)$ of an InP MISFET with a refractory gate $\left(\mathrm{WN}_{x}\right), L_{\mathrm{g}}=5 \mu, W_{\mathrm{g}}=250 \mu$.] 
bulles et de fissures, allant jusqu'au décollement des couches. Les performances sont encore modestes. La transconductance est de $12 \mathrm{mS} / \mathrm{mm}$ pour une longueur de grille de $5 \mu \mathrm{m}$. Cependant la dérive du courant drain-source, qui reste une des pierres d'achoppements de la technologie MISFET, est limitée ici à $4 \%$ sur une période de $10^{4}$ secondes, en régime linéaire $\left(V_{\mathrm{ds}}=0,1 \mathrm{~V}\right.$ et $\left.V_{\mathrm{gs}}=2,5 \mathrm{~V}\right)$ (Fig. 6).

\section{Conclusion.}

Dans cette étude nous avons cherché à déterminer la composition de films de silice déposés par UVCVD. Ils se révèlent être riches en hydrogène sous la forme de $\mathrm{SiOH}$ et $\mathrm{H}_{2} \mathrm{O}$. Les conditions de fabrication permettent d'éliminer des spectres infrarouges les absorptions de $\mathrm{SiH}$, et $\mathrm{N}=\mathrm{O}$. Le recuit rapide à haute température permet l'évaporation de la majorité de ces espèces, ainsi qu'une amélioration de la résistivité. Les défauts créés par ce type de recuit, apparition de charges fixes sans création de liaisons $\mathrm{Si}-\mathrm{O}$, peuvent être guéris par un recuit à basse température, avec formation de liaisons manquantes. Les premiers composants montrent la faisabilité d'une telle technologie malgré la fragilité du matériau. Les composants autoalignés semblent obtenir des résultats prometteurs sur la maîtrise de la dérive du courant source-drain [5].

\section{Remerciements.}

Les auteurs tiennent à remercier A. Falcou, P. Krauz et $\mathrm{A}$. Madouri pour leurs contributions à ce travail.

\section{Bibliographie}

[1] Pliskin W. A. and Lehman H. S., Structural evaluation of silicon oxide films, J. Electrochem. Soc. 112 (1965) 1013.

[2] Dimitriou P., Courant J. L. and Post G., Low temperature photon assisted $\mathrm{CVD}$ of $\mathrm{SiO}_{2}$ on InP, proceedings INFOS 89 (Garching, RFA).

[3] PIEl J. P., Caractérisation de structures MIS par ellipsométrie spectroscopique, Thèse de l'Université de Caen (1987).
[4] Pliskin W. A., Comparison of properties of dielectrics films deposited by various methods, J. Vac. Sci. Technol. 14 (1977) 1064.

[5] Gardner P. D. et al., The InP/low temperature deposited $\mathrm{SiO}_{2}$ interface, Proceedings First internat. Conf. on InP and rel. materials (Norman, Oklahoma) à paraître. 